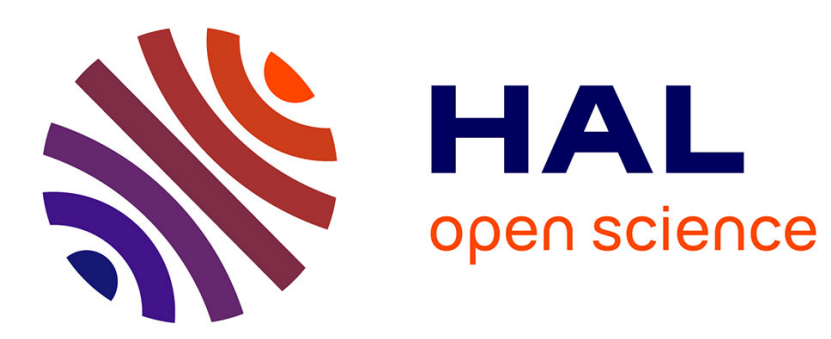

\title{
Molecular simulations of grafted metal-chelating monolayers: methodology, structure and energy
}

Florent Goujon, Christine Bonal, Benoit Limoges, Patrice Malfreyt

\section{To cite this version:}

Florent Goujon, Christine Bonal, Benoit Limoges, Patrice Malfreyt. Molecular simulations of grafted metal-chelating monolayers: methodology, structure and energy. Molecular Physics, 2008, 106 (11), pp.1397-1411. 10.1080/00268970802235557 . hal-00513214

\section{HAL Id: hal-00513214 \\ https://hal.science/hal-00513214}

Submitted on 1 Sep 2010

HAL is a multi-disciplinary open access archive for the deposit and dissemination of scientific research documents, whether they are published or not. The documents may come from teaching and research institutions in France or abroad, or from public or private research centers.
L'archive ouverte pluridisciplinaire $\mathbf{H A L}$, est destinée au dépôt et à la diffusion de documents scientifiques de niveau recherche, publiés ou non, émanant des établissements d'enseignement et de recherche français ou étrangers, des laboratoires publics ou privés. 


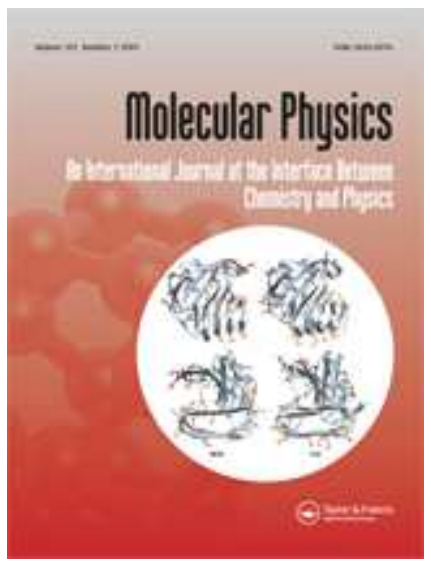

Molecular simulations of grafted metal-chelating monolayers: methodology, structure and energy

\begin{tabular}{|c|c|}
\hline Journal: & Molecular Physics \\
\hline Manuscript ID: & TMPH-2008-0087.R1 \\
\hline Manuscript Type: & Full Paper \\
\hline $\begin{array}{r}\text { Date Submitted by the } \\
\text { Author: }\end{array}$ & 28-May-2008 \\
\hline Complete List of Authors: & $\begin{array}{l}\text { Goujon, Florent; Laboratoire de Thermodynamique et Interactions } \\
\text { Moléculaires, Chemistry } \\
\text { Bonal, Christine; Laboratoire de Thermodynamique et Interactions } \\
\text { Moléculaires, Chemistry } \\
\text { Limoges, Benoit; Laboratoire d'Electrochimie Moléculaire, chemistry } \\
\text { MALFREYT, Patrice; Blaise Pascal University, Chemistry }\end{array}$ \\
\hline Keywords: & $\begin{array}{l}\text { molecular dynamics, long range electrostatic interactions, } \\
\text { monolayers, grafted surface, methodology }\end{array}$ \\
\hline
\end{tabular}

\section{scholarONE" \\ Manuscript Central}


Molecular Physics, Vol. 00, No. 00, DD Month 200x, 1-32

\title{
Molecular simulations of grafted metal-chelating monolayers: methodology, structure and energy.
}

\author{
F. Goujon ${ }^{1}$, C. Bonal ${ }^{1}$, B. Limoges ${ }^{2}$ and P. Malfreyt ${ }^{1 *}$ \\ ${ }^{1}$ Laboratoire de Thermodynamique et Interactions Moléculaires, FRE 3099, Université Blaise Pascal, 63177 \\ Aubière Cedex, France \\ ${ }^{2}$ Laboratoire d'Electrochimie Moléculaire, UMR CNRS 7591, Université Paris Diderot, (Paris VII), 2, Place \\ Jussieu, 75251 Paris Cedex 05, France
}

(v2.0 May 2008)

\begin{abstract}
:
We report molecular dynamics simulations of monolayers of copper complexes covalently attached to a graphite surface in water. Simulations are performed to calculate the structural (density profiles, distribution functions), dynamical (diffusion coefficients) and energetical properties of typical systems presenting a finite length in the third dimension. The results are discussed as a function of the method used for computing the long-range Coulombic interactions. Three different methodologies are used: a two dimensional method (HKE) more extensively in simulation time, the standard Ewald summation technique (EW3D) and the corrected Ewald method (EW3DC). From a computational viewpoint, the comparison between methods is essential to establish the set-up conditions and it represents a pre-requisite work before further investigating these grafted systems. We complete this study by providing a molecular description (structure, energy, diffusion, hydration) of the grafted monolayers of copper complexes and of the water molecules.
\end{abstract}

\footnotetext{
*Corresponding author. Email: Patrice.MALFREYT@univ-bpclermont.fr

Molecular Physics

ISSN 0026-8976 print/ ISSN 1362-3028 online (C)2007 Taylor \& Francis Ltd

http://www.tandf.co.uk/journals

DOI: $10.1080 / 002689700 \mathrm{xxxxxxxxxxxx}$
} 


\section{Introduction}

Monolayers of organic molecules grafted onto solid surfaces have been the focus of intense research because of their potential use in a plethora of technological applications ranging from biosensing applications to microfabrication technology [1]. One of the most investigated monomolecular layer on solid surfaces are the self-assembled monolayers formed via strong chemisorption of alkanethiols on gold surfaces [2-5]. An important aspect of such interfacial engineering is to control the organization, orientation, and functional properties of immobilized molecules at the monolayer. This is particularly important for the immobilization of proteins that are relatively sensitive to unfolding, steric hindrance and inactivation on surface. Consequently, during the last years, considerable efforts has been done to develop new immobilization strategies that circumvent the problem of biomolecules inactivation on surfaces. Among these strategies, the method based on metal-ion chelating agents and their specific affinity for proteins containing histidine residues was proved particularly useful to control protein orientation on surfaces and to preserve their function [6]. In this system, the imidazole moieties of the protein can chelate to the free coordination sites of divalent transition-metal ions such as $\mathrm{Cu}(\mathrm{II})$, which are themselves immobilized onto solid supports via appropriate chelating agents such as the tetradentate nitrilotriacetic acid (NTA) ligand. Self-assembled monolayers of thiol-functionalized-NTA on gold supports have been the most widely investigated. However, the use of gold as solid support presents a number of disadvantages such as the oxygen sensitivity of thiol compounds, the moderate stability of the self-assembled monolayer upon storage. To overcome some of these problems, carbon surfaces were recently functionalized by self-assembled monolayers of NTA-modified loaded vith $\mathrm{Cu}(\mathrm{II})$ [7] (Figure 1).

A better understanding of the general properties governing these monolayers of metal-chelating ligands attached to electrodes is required. In fact, interfacial self-assembled monolayers properties are determined by molecular structure on the surface which is strongly affected by molecular intra-and inter chain interactions and interactions with solvent as well as with the substrate [8,9]. It also appears that there is still a dire need for probes capable of providing structural information to know the details of molecular-scale surface architecture. 
Computer simulation has already made important contribution to the understanding of the processes involved about molecules and reactions and is now a vital adjunct to experimental studies [10]. Molecular simulation employ force field functions that calculate the overall potential energy of a system based on a summation of individual atom-atom pair interactions. The force field equations take into account the contributions from binding interactions and non-binding interactions. Following the pioneering work of Klein's group [11,12], a number of simulations have employed force field to explore the structure and dynamics of self-assembled monolayer (SAM). However, the majority of the work has been limited to the study of alkanethiols of different tail-groups which self assembled on Au surface in the presence of water [11-21]. Several molecular dynamics and Monte Carlo computer simulations studies of carboxylic acid-functionalized self-assembled monolayers [22-24] and of oligo(ethylene oxide) (OEO) or poly(ethylene oxide) (PEO) self-assembled monolayers have also recently been reported [25-27]. However, very few theoretical chemical studies of monolayers on others surfaces that gold have been reported [28-31] and none of them have been focused on negatively charged monolayers of chelating metal compounds immobilized onto carbon surface via the tetradentate nitrilotriacetic acid.

The presence of a surface creates a nonuniformity of the local density along the direction normal to the surface and gives rise to important issues concerning the truncation procedures and the methodology used to account the Coulombic interactions. Additionally, this type of system is represented by a slab geometry in molecular simulations where periodic boundary conditions are used in two of the three directions and physical boundaries preventing molecules from leaving the primary cell. For this slab geometry, the calculation of the long-range Coulombic interactions cannot be performed directly using the standard Ewald summation method $[32,33]$ which is the most widely applied technique for this kind of interactions in a three-dimensionally periodic system. Different methodologies can be thus established. Some of them [34-36] are designed to calculate long-range interactions in systems periodic in two directions but bound in the third one. The Hautmann-Klein Ewald method (HKE) [35] is one of these methods and corresponds to an adaptation of the Ewald method for systems which are periodic in two dimensions only. Another alternative consists of applying the standard three dimensional Ewald summation method (EW3D) to a simulation box elongated in the $z$ direction so that a sufficiently large empty space is left between the periodic images $[37,38]$ to dampen out the interslab interactions. A more elaborated 
treatment of the long-range interactions (EW3DC method) consists of modifying the EW3D method by the addition of a correction term to remove the total dipole moment of periodically repeating slabs.

It is essential from a computational viewpoint to compare the different alternatives to estimate the long-range coulombic interactions. The HKE method is in fact well adapted for slab-like systems however, it is very time consuming with a simulation time 10-20 times higher than that of EW3D. Two reference systems are then studied using either one two-dimensional method (the Hautman-Klein method) or two three dimensional methods (EW3D, EW3DC). These reference systems are built in such a way that the size of the system allows the comparison in a reasonable simulation time. We then perform a series of comparisons on the structural, dynamical and energetical properties. The first reference system is constituted by only water molecules embedded between two parallel graphite plates. We have then extended the comparison on a second reference system of our main interest which concerns the negatively charged monolayers of chelating copper compounds immobilized onto a graphite surface in an aqueous solution. Finally, we apply the EW3DC methodology to obtain a molecular description on the structure, hydration, dynamics and energetic properties of a more sophisticated system of these monolayers.

The outline of the paper is as follows. In section 2, we present the potential model with the different methods for calculating the long-range Coulombic interactions. In section 3 , we discuss the results as a function of the method used by paying attention on the system formed by monolayers of charged copper complexes covalently attached to a graphite surface in water. We focus on the electrical, structural and diffusion properties on the grafted NTA-Cu(II) ligands using the EW3DC method. We conclude in section 4 by a brief summary of our main results.

\section{Computational procedures}

\section{$2.1 \quad$ Model}

In the case of the modelling of the NTA molecule, we use the all-atom (AA) version of the Cornell force field AMBER [39]. The general potential function is of the form 


$$
\begin{aligned}
U & =\sum_{\text {bonds }} k_{b}\left(r-r_{o}\right)^{2} \\
& +\sum_{\text {angles }} k_{\theta}\left(\theta-\theta_{o}\right)^{2} \\
& +\sum_{\text {dihedrals }} k_{\phi}[1+\cos (l \phi+\delta)] \\
& +\sum_{i=1}^{N-1} \sum_{j=i+1}^{N}\left\{4 \epsilon_{i j}\left[\left(\frac{\sigma_{i j}}{r_{i j}}\right)^{12}-\left(\frac{\sigma_{i j}}{r_{i j}}\right)^{6}\right]+\sum_{l}^{\infty} \frac{q_{i} q_{j}}{\left|\mathbf{r}_{\mathbf{i j}}+\mathbf{n} L\right|}\right\}
\end{aligned}
$$

where $k_{b}, k_{\theta}$ and $k_{\phi}$ are the force constants for deformation of bonds, angles and dihedrals, respectively. The equilibrium values of bond distances and valence angles correspond to $r_{o}$ and $\theta_{o}$, respectively. In the dihedral angle term, $l$ is the periodicity and $\delta$ is the phase factor. The $\mathrm{C}-\mathrm{H}$ and $\mathrm{O}-\mathrm{H}$ covalent bonds were kept of fixed length by use of the SHAKE algorithm [40] and the aromatic rings were kept planar using six improper torsional potentials. The intermolecular and intramolecular interactions consist of a van der Waals repulsion-dispersion term calculated using the Lennard-Jones (6-12) potential, represented by the penultimate term in Eq.(1). In the AMBER force field, the nonbonded interactions between atoms separated by exactly three bonds (1-4 van der Waals interactions) are reduced by a factor of 0.5 [39]. The Lennard Jones potential parameters for the interactions between unlike atoms were calculated by using the Lorentz-Berthelot mixing rules (quadratic and arithmetic rules for $\epsilon_{i j}$ and $\sigma_{i j}$ parameters, respectively). The water molecules were represented with the TIP4P/2005 model [41]. This representation (Table 1) consists of four interaction sites: three of them are placed at the oxygen and hydrogen atom positions, respectively. The fourth site is placed at the bisector of the $\mathrm{H}-\mathrm{O}-\mathrm{H}$ angle.

The metal-ligand interactions are treated as being fully covalent and are modeled using the Morse potential [42].

\section{$2.2 \quad$ Long-range electrostatic interactions}

The last term in Eq.(1) corresponds to the electrostatic energy $\left(E_{E L E C}\right)$ of the system. Consider $N$ molecules in a volume $V=L_{x} L_{y} L_{z}$ with center of mass $\mathbf{r}_{\mathbf{i}}$, each molecule contains $n_{i}$ charges $q_{i a}$ at position $\mathbf{r}_{\mathbf{i a}}$. The electrostatics interactions handled with the Ewald sum method $[32,43]$ in a box with 
orthogonal axis that is overall charge neutral are given by the following contributions

$$
\begin{aligned}
U_{E L E C} & =\frac{1}{2 \epsilon_{o} V} \sum_{k \neq 0} Q(h) S(\mathbf{h}) S(-\mathbf{h}) \\
& +\frac{1}{8 \pi \epsilon_{o}} \sum_{i} \sum_{a} \sum_{j \neq i} q_{i a} \sum_{b} q_{j b} \operatorname{erfc}\left(\alpha r_{i a j b} / r_{i a j b}\right) \\
& -\frac{\alpha}{4 \pi^{3 / 2} \epsilon_{o}} \sum_{i} \sum_{a} q_{i a}^{2} \\
& -\frac{1}{8 \pi \epsilon_{o}} \sum_{i} \sum_{a} \sum_{b \neq a} \frac{q_{i a} q_{i b}}{r_{i a i b}} \operatorname{erf}\left(\alpha r_{i a i b}\right) \\
& +\frac{1}{2 \epsilon_{o} V} M_{z}^{2}
\end{aligned}
$$

where $\operatorname{erfc}(x)$ is the complementary error function and $\operatorname{erf}(x)$ is the error function. $\alpha$ is chosen so that only pair interactions in the central cell need to be considered in evaluating the second term in Eq.(2). In the last term of Eq.(2), $M_{z}$ is the net dipole moment of the simulation cell given by $\sum_{i=1}^{N} q_{i} \mathbf{r}_{i}$. This contribution is the correction term from Yeh and Berkowitz [37] which results by the plane-wise summation method proposed by Smith [43]. Adding this term to the total energy amount to using a $z$-component force for each atom given by

$$
F_{i, z}=-\frac{q_{i}}{\epsilon_{o} V} M_{z}
$$

The EW3DC method differs from the standard EW3D method only by the presence of this dipole correction. The functions $S(\mathbf{h})$ and $Q(h)$ are defined using the Eqs.(4) and (5), respectively

$$
\begin{gathered}
S(\mathbf{h})=\sum_{i} \sum_{a} q_{i a} \exp \left(i \mathbf{h} . \mathbf{r}_{\mathbf{i a}}\right) \\
Q(h)=\frac{1}{h^{2}} \exp \left(-\frac{h^{2}}{4 \alpha^{2}}\right)
\end{gathered}
$$

where the reciprocal lattice vector $\mathbf{h}$ is defined as $\mathbf{h}=2 \pi\left(l / L_{x}, m / L_{y}, n / L_{z}\right)$ where $l, m, n$ take values of $0, \pm 1, \pm 2, \cdots \pm \infty$. The reciprocal space sum is truncated at an ellipsoidal boundary at the vector $\left|\mathbf{h}^{\max }\right|$. The values of the components of the vector $\left|\mathbf{h}^{\max }\right|$ are given in Table 1 for each system. 


\section{$2.3 \quad$ Set-up conditions}

The equations of motions are integrated using the Verlet Leapfrog algorithm scheme at $\mathrm{T}=298 \mathrm{~K}$ with 2 fs as timestep. The simulations are performed in the NVT ensemble using the Hoover algorithm [51] with a coupling constant of $0.5 \mathrm{ps}$. SHAKE algorithm is used to preserve the rigidity of the water molecules and the $\mathrm{OH}$ and $\mathrm{CH}$ bonds in the NTA molecule. The cutoff for the Lennard-Jones contribution is fixed to $12 \AA$ whereas it is equal to $18 \AA$ for the real space of the electrostatic interactions. We also use a reciprocal space cutoff radius of $1.14 \AA^{-1}$. The box dimensions, the number of water molecules, the Ewald parameters are reported in Table 1 as a function of the system. The configurations are generated using the parallel version of the modified DL_POLY_MD package [52] by using up to 8 processors at a time. The parameters of the long-range electrostatics interactions evaluated by either the Ewald summation technique or the HKE techniques have been calculated in order to satisfy a relative error of $10^{-6}$. In the case of water embedded between two parallel plates, a run consisted of 200 ps of equilibration followed by a production phase of additional $600 \mathrm{ps}$. To allow a possible comparison between the different methods in a reasonable simulation time, an equilibration period of 200 ps and an acquisition phase of 300 ps were performed over the NTA-Cu(II) grafted surfaces. The corresponding CPU times are reported in Table 1 for comparison. 


\section{$3 \quad$ Results and discussions}

\subsection{Comparison between HKE, EW3D and EW3DC methods}

Firstly, a system formed by 2340 water molecules embedded between two parallel graphite plates was studied. This latter has the particularity to present the total dipole moment of the simulation cell close to zero. It is then expected that the EW3D and EW3DC methods give the same results. A series of molecular dynamics simulations of liquid water between two parallel graphite plates with no defects with $\mathrm{C}-\mathrm{C}$ distance of $1.42 \AA$ have been performed at room temperature $(\mathrm{T}=298 \mathrm{~K})$ and fixed densities. The graphite surface is composed by one layer of $16 \times 18$ unit cells leading to 576 carbon atoms and to an area of $\left(39.4 \times 38.3 \mathrm{~A}^{2}\right)$. The carbon atoms of the graphite layer are treated as fixed on the initial rigid lattice. We have also described the graphite as a set two or three flat sheets separated by $3.35 \AA$ and stacked perpendicularly to the $z$ axis. We have observed no difference on the structural and energetical properties of the system when the number of layers is increased. As a result, the graphite surface is modelled as only one flat sheet in the present work. The system is thus composed of two sheets of highly oriented pyrolytic graphite (HOPG) separated by a distance $d$ of $50 \AA$. The intersurface spacing $d$ is adjusted to yield the bulk density across the simulation box. When we apply the HKE method for the calculation of the long-range Coulombic interactions, the $z$-dimension of the box is fixed to $50 \AA$. We extend this box length dimension up to $190 \AA$ in the case of the calculation of the electrostatic interactions using the EW3D and EW3DC methods to be in line with the recommendations of Spohr [53]. Molecular simulations have been already reported for this system in the literature $[54,55]$. However, our simulations differ by a greater separation distance and a systematic comparison between HKE, EW3D and EW3DC methods.

Figure 2a shows the molecular density profiles of water along the $z$-direction normal to the surfaces. We observe that the profiles can be considered as identical for the three methods and that a region of bulk liquid of approximately $25 \AA$ is formed in the middle of the cell. Part b of Figure 2 shows the molecular temperature profiles of the local temperature $T$ defined by $1 / 3\left(T_{x x}+T_{y y}+T_{z z}\right)$ components. The $T_{\alpha \beta}$ component along the $z$-direction is calculated via Eq.(6) 


$$
k_{B} T_{\alpha \beta}(z)=\left\langle\frac{\sum_{i=1}^{N} H\left(z_{i}\right) m_{i}\left(\mathbf{v}_{\mathbf{i}}\right)_{\alpha} \cdot\left(\mathbf{v}_{\mathbf{i}}\right)_{\beta}}{\sum_{i=1}^{N} H\left(z_{i}\right)}\right\rangle
$$

$$
H\left(z_{i}\right)=\left\{\begin{array}{l}
1 \text { for } z-\frac{\Delta z}{2}<z_{i}<z+\frac{\Delta z}{2} \\
0 \text { otherwise }
\end{array}\right.
$$

where $\Delta z$ is the slab thickness. The analysis of the temperature profile establishes that the temperature is identical for each $z$ and equal to the input temperature for EW3D and EW3DC. For HKE, we observe a slight decrease of the local temperature in the middle of the box (deviation of $1 \%$ from the input $T$ ) whereas there are slight increases of $T$ near the surfaces with the same deviation. However, the average temperature calculated over the slabs is $300 \pm 3 \mathrm{~K}$ for HKE and $300 \pm 1 \mathrm{~K}$ for EW3D and EW3DC. We also check that the diagonal elements of the temperature tensor are equivalent showing an identical equipartition of the kinetic energy in the three directions for all the methods. The profiles of the off-diagonal elements of the temperature tensor fluctuate about a zero mean value, as expected for a system at thermal equilibrium.

We examine the structure of water in Figure 2c by calculating the oxygen-oxygen pair correlation function in $1.0 \AA$ slab centered in the middle of the box for the three methods. We add for comparison the three dimensional oxygen-oxygen radial distribution function calculated in a bulk liquid simulation box. The fact that the plots resulting from the two-dimensionally periodic systems are identical with that of the three-dimensionally periodic bulk water system confirm that we have a region of bulk water in the middle of the cell. We also check that the structure of water is described in the same way with HKE, EW3D and EW3DC. To complete the comparison between the methods on this system, it is very interesting to compare the degree to which the water molecules are correlated over long distances. This can be done by 
calculating the finite-system $g$-Kirkwood factor [56] $G_{k}$ from the following equation

$$
G_{k}=\frac{\left\langle\mathbf{M}^{2}\right\rangle-\langle\mathbf{M}\rangle\langle\mathbf{M}\rangle}{N \mu^{2}}
$$

where $\mathbf{M}$ is the total dipole moment of the simulation box and $\mu^{2}$ is the mean-squared molecular dipole moment. For the TIP4P/2005 model, $\mu$ is equal to 2.305 D. Figure 3a displays the cumulative time average of the factor $G_{k}$ over $600 \mathrm{ps}$. We check that $G_{k}$ is a slowly fluctuating property and that it requires significant simulation time to converge. We obtain final values of $G_{k}$ of 2.2, 2.2 and 2.1 for HKE, EW3D and EW3DC, respectively. For comparison, $G_{k}$ is equal to 3.1 for bulk water system. We show that the correlation of water molecules over long distances where long-range electrostatic continue on playing a major role does not depend on the method used (KHE, EW3D, EW3DC) in the case of water embedded between two graphite plates. We also check that the cumulative time average of the $\langle\mathbf{M}\rangle\langle\mathbf{M}\rangle$ quantity (Figure $3 b)$ converges to zero at long time. This quantity measures the absolute values of the fluctuations and is a good parameter to establish the convergence of $M$. We find that the total dipole moment $M$ is equal to zero in agreement with the fact that there is not net polarisation in the simulation cell. This is confirmed by the profile of the $z$-component of $M$ across the simulation cell shown in the inset of Figure 3b. The inset shows that the total $M_{z}$ is zero for each slab and for all the methods. Table 2 reports the total energy contributions of the simulation cell for the Lennard-Jones and electrostatic contributions. We observe that the energy contributions can be considered as similar within the errors bars indicating a equal repartition of the different contributions for the three methodologies. The fact that the values of the Coulombic interactions match perfectly for the three methods also contributes to validate the EW3D methodologies for the calculation of these long range interactions.

We confirm the good agreement between the methods by the calculation of the parallel and perpendicular components of the diffusion coefficient of water by using the Einstein expression [57]. The values of the diffusion coefficient are listed in Table 2 as a function of the methodology. We observe that the different values of the components match satisfactory between HKE, EW3D and EW3DC. We also check that the components calculated from the Green-Kubo expression [57] are in line with those reported in Table 2 within the statistical uncertainties. In the case of separation distance of $50 \mathrm{~A}$, a large percentage of water molecule is far from the surfaces suggesting that the influence of the surfaces is reduced. 
This explains why we find values of diffusion coefficient close to that calculated in bulk water with the TIP4P/2005 model $\left(2.4 \times 10^{-5} \mathrm{~cm}^{2} \mathrm{~s}^{-1}\right)$ and to the experimental self-diffusion coefficient of water [58] at $298 \mathrm{~K}\left(2.3 \times 10^{-5} \mathrm{~cm}^{2} \mathrm{~s}^{-1}\right)$. This assertion can be checked through the analysis of the perpendicular component $D_{\perp}$ which does not differ significantly from the parallel component.

We now focus of a system consisting in grafting randomly 10 NTA-Cu(II) complexes onto a HOPG graphite surface formed by 576 atoms, the surface coverage being then $110 \mathrm{pmol} \mathrm{cm}{ }^{-2}$ [7]. Each chain is covalently attached to a carbon of the graphite surface (Figure 1). A second graphite surface bounds the simulation in the $z$-direction. We take the route of distributing the total positive charge $(+10$ e) on each carbon atom with a charge of (10/576 e) of the second graphite surface to obtain a neutral system. This method avoids both the addition of counter-ions and the increase of the box size along the $z$ axis. We have chosen this system model in order to have a system size allowing a comparison in a reasonable simulation time between the two-dimensional (HKE) and three dimensional Ewald methods. Whereas $M_{z}$ is equal to $0 \mathrm{D}$ in the case of water embedded between two graphite plates, $M_{z}$ is about - $0.6 \mathrm{D}$ for the grafted system.

Figure 4a presents the density profiles of the water molecules and of the grafted NTA-Cu(II) complexes for the three methodologies. Interestingly, we observe that the different methods yield the same density profiles. The profile of the number of hydrogen bond per water molecules is reported in Figure 4b. Let us recall that two water molecules are identified as being hydrogen bonded only if the oxygen-oxygen distance is less than $3.5 \AA$ and simultaneously the $\mathrm{OH} . . \mathrm{O}$ distance is less than $2.45 \AA$. We check that respecting these two conditions leads to the oxygen-oxygen-hydrogen angle is less than $30^{\circ}$ [59-61]. The profile shows a decrease in the number of hydrogen bonds until $z=16 \AA$. Up to $16 \AA$, the number of hydrogen bonds increases again. The fact that the profiles of the number of hydrogen bond per water molecules and the oxygen-oxygen pair correlation function in the bulklike (Figure $4 \mathrm{c}$ ) are very close for HKE, EW3D and EW3DC indicate that the structure of the water is correctly described within the system. Finally, all structural properties studied here show that the three-dimensional methods give results in perfect accordance with the two-dimensional HKE method. Let us consider results obtained for energetical and dynamical properties (Table 2). The values of the Lennard-Jones and Coulombic interactions deviate from less than $1 \%$ between the three methods. We also observe that the diffusion 
coefficients calculated at two different locations match satisfactory between HKE, EW3D and EW3DC. The components of the diffusion coefficients calculated in zone 1 defined by $25 \AA<z<50 \AA$ are in fact very close to those of the water embedded between graphite planes. In the case of zone 2 defined by 0 $\AA<z<25 \AA$, water molecules strongly interact both with the grafted molecules to give preferential hydrogen bonds and with the graphite surface involving preferential orientations. This leads to a slower diffusion of the water molecules in the interfacial region.

The fact that the EW3D and EW3DC method lead to the same results for the two studied systems can be explained by the dimensions of the simulation cell that makes an empty space length five times larger than the space of the primary occupied cell. Using such box dimensions, the methods are made very close. However, from a theoretical viewpoint, it is more rigorous to use the EW3DC method.

\section{$3.2 \quad$ Structure and energy}

Given that the EW3DC methodology correctly reproduces the results of a two-dimensional methods, we apply then it one a more sophisiticated system of grafted NTA-Cu(II) system. In fact, we decide to improve our methodology by taking into account the presence of counter-ions in solution to counterbalance the negative charges of the grafted complexes and to better model the experimental conditions. We then add to the simulation 10 mobile $\mathrm{Na}^{+}$cations to obtain a neutral system. We extend then the primary $z$-dimension from $50 \AA$ to $80 \AA$ A. It amounts to extending within the EW3DC methodology the total $z$-dimension from to $190 \AA$ to $320 \AA$ with two empty spaces of $120 \AA$ surrounding the primary cell. The different parameters for this simulation are given in Table 1. The simulation time is increased up to $1 \mathrm{~ns}$ for the production phase. The previous methodology consisting in charging the opposite graphite surface leads to the creation of dielectric field across the direction normal to the surfaces. In the case of the addition of counter-ions, we observe in Figure 5a, a quasi-zero profile of the local $M_{z}(z)$ across the $z$-direction from $z=60 \AA$. This indicates that the $z$-dimension is developed enough to yield a bulklike region from the grafted surface. To further investigate this behavior, we have plotted in part b of Figure 5 the angular density of probability $n(\cos \theta)$ as a function of $\cos \theta$ for three different zones A, B and C. $\theta$ is the angle between the molecular dipole moment vector of water molecules and the constant unit vector normal to the graphite surface. We 
observe an uniform distribution of $\cos \theta$ for water in zone $\mathrm{C}$ as expected for distributions in bulklike region. Zone A, which is close to the ends of grafted molecules, is characterized by a preferential orientation of water molecules with respect to the graphite surfaces: the hydrogen atoms of water molecules point to the surface. This effect is much less marked for the intermediate region B and disappears for zone A. To check the influence of the counter-ions on the electrical properties of the system, we calculate the charge density profile across the simulation box from the following expression

$$
\rho_{q}(z)=\sum_{i} q_{i} \rho_{i}(z)
$$

where $q_{i}$ is the charge of the atom $i$ and $\rho_{i}(z)$ is the density number profile. The profile of the electric field $E(z)$ along the $z$ direction can be calculated by integrating the charge density profile according to

$$
E(z)=\frac{1}{\epsilon_{0}} \int_{-\infty}^{z} \rho_{q}\left(z^{\prime}\right) d z^{\prime}
$$

where $\epsilon_{0}$ is the vacuum permittivity. As expected from the nonisotropic distribution of the water dipole moment at the surface, the charge density profile shown in Figure $5 \mathrm{c}$ does not vanish close to the surface with strong fluctuations at distances closer than $10 \AA$ from the graphite surface. From a distance greater than $20 \AA$, the charge density profile is zero. The analysis of the electric field profile in Figure $5 \mathrm{~d}$ shows that the electric field reaches a constant zero value from $20 \AA$ and confirms the fact that the presence of the counter-ions does not prevent the development of a fully bulklike region in the centre of the simulation box with an isotropic distribution of the water molecules. These electrical features could be recovered in the use of oppositely charged surfaces as long as there is sufficient separation distance between the surfaces.

We have shown that the use of the EW3DC methodology and the explicit modelling of the counter-ions produce consistent description of the electrical and thermodynamic properties of the grafted NTA-Cu(II) system. These checks are essential before an extensive study of the energetical and structural properties of the system. Our conclusions concerning the use of the EW3DC method in interfacial systems which are non-periodic in one of the three directions are in line with those drawn from previous works [37,62-64]. 
14

We now focus on the description of the physical insights of the grafted NTA-Cu(II) system. A typical snapshot of the system with the water molecules is shown in Figure 6 in the case of a surface coverage of $110 \mathrm{pmol} \mathrm{cm}^{-2}$. A detailed description of the modified graphite surface requires to represent the density profiles of atoms of the grafted molecules as a function of the distance from the surface. We represent in Figure 7a the density profiles of several atoms such as of the oxygen atoms of the carboxylate end groups, the copper and sodium atoms. We also report the profile of the carbon atom of the amide group close to the phenyl group covalently attached to the graphite surface. The profiles of the oxygen atoms of the carboxylate groups extend over a region of $13 \AA(5 \AA<z<18 \AA)$ indicating a high mobility of the terminal group of the grafted molecules. The profile of the amide carbon shows a well-defined narrow peak indicating that the first part of the molecule is rigid with very little degrees of freedom due to the covalent bond between the aryl group and the graphite surface. As expected from the modelling of bonds between the copper cation and some oxygen atoms of the terminal carboxylate groups, the profile of the copper ion is found to be similar to that of the carboxylate groups. The counter-ions present an uniform profile in the zone of $5 \AA<z<20 \AA$, in line with the fact that these regions are occupied by the polar groups of the grafted molecules. We also note that a very weak proportion of counter-ions can diffuse far from the surface but they do not affect the electrical properties of these zones. Figure $7 \mathrm{~b}$ shows the density profiles of hydrogen bonds between the carboxylate end groups and water molecules and between the amide group and the water molecules. Hydrogen bonds can exist between the grafted molecules and water molecules within the region $4 \AA<z<20 \AA$. The inset of Figure $7 \mathrm{~b}$ shows the total density of water-water hydrogen bonds and water-grafted molecules hydrogen bonds. We observe in the resulting profile that the value of $0.10 \mathrm{gcm}^{-3}$ obtained in the bulklike zone is not recovered in the zone occupied by the grafted molecules. This means that the number of hydrogen bonds between the grafted molecules and water do not compensate the loss of hydrogen bonds between water molecules due to grafting of NTA-Cu(II) complexes.

Figure 8a displays the mean squared displacements of the molecular centres of water molecules in the parallel and perpendicular directions for the grafted zone $(0 \AA<z<20 \AA)$ and the bulklike zone $(35 \AA$ $<z<80 \AA$ ). We give for comparison the mean squared displacements of the molecular centres of water in the case of isotropic bulk water. In the case of the grafted system, we take into account only the water molecules which have never left the region of interest during the calculation. The parallel component 
of the diffusion coefficient in the grafted and bulklike regions is equal to $1.0 \times 10^{-5} \mathrm{~cm}^{2} \mathrm{~s}^{-1}$ and to $2.4 \times$ $10^{-5} \mathrm{~cm}^{2} \mathrm{~s}^{-1}$, respectively, whereas the perpendicular component decreases from $2.0 \times 10^{-5} \mathrm{~cm}^{2} \mathrm{~s}^{-1}$ in the bulk region to $0.8 \times 10^{-5} \mathrm{~cm}^{2} \mathrm{~s}^{-1}$ in the region close to the surface. In the bulklike region, the value of the lateral component of the diffusion coefficient is equal to that of the isotropic diffusion coefficient for the TIP4P/2005 water model at $298 \mathrm{~K}\left(2.4 \times 10^{-5} \mathrm{~cm}^{2} \mathrm{~s}^{-1}\right)$ whereas the value of the perpendicular component is slightly weaker. This indicates that the diffusive behaviour of water molecules in the $z$ direction ranging from $35 \AA<z<80 \AA$ is weakly retarded due to the presence of the graphite surface. However, Figure $8 \mathrm{~b}$ shows that the normalized velocity autocorrelation functions of water molecules in the bulklike region match very well with that of the isotropic bulk water and tend slowly to zero. The lateral and perpendicular components of the velocity correlations functions calculated in the grafted zone contrast with those calculated in the bulk region by a more negative first peak and by the fact that these curves tend to zero with damped oscillations. These oscillations mean that the water molecules are trapped in a cage formed by neighbouring water molecules and NTA-Cu(II) grafted ligands. The fact that the first oscillations appear for negative values of the correlation functions indicate that the reversal of the velocities is correlated and that the cage effect has a certain lifetime. From 0.6 ps, the correlation functions oscillate around zero indicating that uncorrelated cages are formed successively.

The energetic description of the system requires to calculate different energy contributions between grafted molecules and between grafted molecules and the water molecules. We report in Figure 9a the average values of the dispersive contribution to the Lennard-Jones potential and of the electrostatic energy between a given molecule and the rest of the grafted molecules as a function of the molecule number. The dispersive term modelled as $-\frac{1}{r^{6}}$ is used to model the van der Waals interactions whereas the $-\frac{1}{r^{12}}$ represents the repulsive interactions. We observe an inhomogeneous distribution of the dispersive term as a function of the grafted molecule number whereas the distribution of the electrostatic contribution is much more homogeneous. The dispersive and repulsive terms of the Lennard-Jones potential give important contributions at short distances; the electrostatic contribution in $\left(\frac{1}{r}\right)$ continue to yield contributions at larger distances. It means that the repulsive and dispersive terms are very sensitive on the way of grafting the molecules and of the local environment whereas the electrostatic contribution gives rather an average contribution for the grafted surface. Grafting NTA-Cu(II) ligands amounts to constraining molecules along 
the direction normal to the surface; these molecules cannot relax to reduce the repulsion between their partial charges. This leads to an unfavorable electrostatic energy contribution. Part b of Figure 9 shows the dispersive and electrostatic energy contributions between each grafted NTA-Cu(II) ligand and the water molecules. We also give for completeness the number of water molecules interacting for each grafted molecule with the Lennard-Jones $\left(r_{c}=12 \AA\right)$ and electrostatic $\left(r_{c}=18 \AA\right)$ cutoffs. Interestingly, we observe that each NTA-Cu(II) molecules interacts approximately with the same number of water molecules. This uniform distribution of the electrostatic and dispersive contributions shows that the hydration of the grafted molecule does not depend on the way of grafting the molecule for this value of surface coverage.

\section{Conclusions}

In view of the modelling of monolayers of copper complexes covalently attached to graphite surface in water, we have compared one two-dimensional (HKE) and two three-dimensional methods (EW3D) and (EW3DC) for the calculation of the long range electrostatic interactions. For a slab geometry, it is necessary to extend enough the primary simulation cell in the $z$-axis so that there is no artificial influence between the periodic images in this direction to use a standard three-dimensional Ewald summation. From a theoretical viewpoint, EW3DC method must be applied when there is a net polarization in the simulation box because it adds a correction term required to remove the leading dipole of the primary cell. We have firstly checked on a typical system formed of water molecules embedded between two graphite surfaces that the density profiles, hydrogen bonds, energy contributions and components of the diffusion coefficient are independent on the method used. We note that this observation is in line with the recommendations given by Yeh and Berkowitz [37] i.e that the current EW3DC method is sufficient in terms of accuracy and efficiently.

Finally, using the same methodology, we have also modelled a grafted system modified in such a way to present a larger $z$-dimension and to contain counter-ions to counterbalance the negative charges of the grafted molecules. The comparison between the different methods were necessary to check some fundamental aspects concerning the thermodynamic equilibria and the electrical properties of the grafted 
system. The fact that the methods compare very well is also very important from a computational viewpoint because the three dimensional methods are 20 times faster than two-dimensional method.

We have then reported a detailed molecular description of the grafted monolayers of copper complexes. We have shown that the carboxylate end groups maintain degrees of freedom and can form hydrogen bonds with the water molecules. The first part of the grafted molecules is strongly constraint with very narrow density profiles due to the covalent bond between the aryl group and the grafted surface. We have shown that the hydrogen bond formed between the grafted molecules and the water cannot compensate the loss of hydrogen bonds between water molecules due to the presence of grafted molecules. The calculation of the diffusion coefficient shows that a bulklike region can also be identified with a slight retard of the diffusion along the normal to the surface. The analysis of the velocity correlation functions in the grafted zone exhibits damped oscillations suggesting a cage effect of water molecules in this zone. The energetic description of the system establishes that the hydration of the grafted molecules is identical for each grafted molecules with a non-homogeneous distribution of the molecule-molecule energy contributions.

Given that the methodology and the system size are now well adapted, we plan to study the impact of some parameters (nature of the surface (gold or graphite), length of the grafted molecule, surface coverage, $\mathrm{pH})$ on the properties of the grafted monolayers. 


\section{References}

[1] G. M. Whitesides, Sci. Am. 9, 146 (1995).

[2] A. Ulman, An Introduction to Ultrathin Organic Films (Academic: Boston, MA, 1991).

[3] C. D. Bain, G. M. Whitesides, Angew. Chem., Int. Ed. 28, 506 (1989).

[4] L. H. Dubois, R. G. Nuzzo, Annu. Rev. Phys. Chem. 43, 437 (1992).

[5] A. Ulman, Chem. Rev. 96, 1533 (1996).

[6] J. Turkova, J. Chromatogr. 722, 11 (1999).

[7] R. Blankespoor, B. Limoges, B. Schöllhorn, J.-L. Syssa-Magalé, D. Yazidi, Langmuir 21, 3362 (2005).

[8] T. Yokoyama, S. Yokoyama, T. Kamikado, Y. Okuno, S. Mashiko, Nature 413, 619 (2001).

[9] M. R. Anderson, M. N. Evaniak, M. Zhang, Langmuir 12, 2327 (1996).

[10] A. R. Leach, Molecular modelling principles and applications, (Pearson Education EMA, 2001).

[11] J. Hautman, M. L. Klein, J. Chem. Phys. 91, 4994 (1989).

[12] J. Hautman, M. L. Klein, J. Chem. Phys. 93, 7483 (1990).

[13] W.Mar, M. L. Klein, Langmuir 10, 188 (1994).

[14] J. Zhang, A. Bilic, R. J. Reimers, S. N.Hush, J. Ulstrup, J. Phys. Chem. B. 109, 15355 (2005).

[15] P. Srivastava, W. G. Chapman, E. P. Laibinis, Langmuir 21, 12171 (2005).

[16] Y.-H. Kim, S. S. Jang, W. A. Goddard, J. Chem. Phys. 122, 244703 (2005).

[17] R. Rousseau, R. Mazarello, S. Scandolo, ChemPhysChem 6, 1756 (2005).

[18] J. Zheng, Y. He, S. Chen, L. Li, M. T. Bernards, S. Jiang, J. chem. Phys. 125, 174714 (2006).

[19] Y.-T. Wang, C.-L. Cheng, Y.-C. Shih, H.-C. Kan, C.-H. Chen, J. J. Hu, Z. Y. Su, C. J. Chem. 25, 1090 (2007).

[20] B. Rai, P. Sathish, C. P. Malhotra, Pradip, K. G. Ayappa, Langmuir 20, 3138 (2004).

[21] R. Mazarello, A. Cossaro, A. Verdini, R. Rousseau, L. Casalis, M. F. Danisman, L. Floreano, S. Scandolo, A. Morgante, G. Scoles, Phys. Rev. Lett. 98, 016102 (2007).

[22] Y. Pei, J. Ma, J. Am. Chem. Soc. 127, 6802 (2005).

[23] J. Lahann, S. Mitragotri, T.-N. Tran, H. Kaido, J. Sundaram, I. S. Choi, S. Hoffer, G. A. Somorjai, R. Langer, Science 299 , 371 (2003).

[24] D. M. Duffy, H. J. Harding, Langmuir 21, 3850 (2005).

[25] E. A. Ismail, G. S. Grest, M. J. Stevens, Langmuir 23, 8508 (2007).

[26] J. A. Pertsin, M. Grunze, Langmuir 16, 8829 (2000).

[27] J. Zheng, L. Li, H. K. Tsao, Y. J. Sheng, S. Chen, S. Jiang, Biophysical J. 89, 158 (2005).

[28] D. S. Kong, S. L. Yuan, Y. X. Sun, Z. Y. Yu, Surface Science 573, 272 (2004).

[29] P. T. Mikulski, A. L. Herman, A. J. Harrison, Langmuir 12197 (2005).

[30] G.Srinivas, S. O. Nielsen, P. B. Moore, M. L. Klein, J. Am. Chem. Soc. 128, 848 (2006).

[31] N. Winter, J. Vieceli, I. Benjamin, J. Phys. Chem. B 112, 227 (2008).

[32] M. P. Allen, D. J. Tildesley, Computer Simulation of Liquids (Clarendon, Oxford University Press, 1981).

[33] D. M. Heyes, Phys. Rev. B 49, 755 (1994)

[34] D. E. Parry, Surf. Sci. 49, 433 (1975).

[35] J. Hautmann, M. L. Klein, Mol. Phys. 75, 379 (1992).

[36] J. Lekner, Physica A157, 826 (1989).

[37] I. C. Yeh, M. L. Berkowitz, J. Chem. Phys. 111, 3155 (1999).

[38] P. S. Crozier, R. L. Rowley, E. Spohr, D. Henderson, J. Chem. Phys. 112, 9253 (2000).

[39] W. D. Cornell, P. Cieplak, C. I. Bayly, I. R. Gould, K. M. Merz, Jr, D. M. Ferguson, D. M. Fox, T. Spellemer, J. W. Caldwell, P. Kolleman, J. Am. Chem. Soc. , 117, 5179 (1995).

[40] J. P. Ryckaert, G. Cicotti, H. J. C. Berendsen, J. Comput. Phys. 23, 327 (1977).

[41] J. L. F. Abascal, C. Vega, J. Chem. Phys. 123, 234505, (2005).

[42] J. Sabolovic, K. R. Liedl, Inorg. Chem. 38, 2764 (1999).

[43] E. R. Smith, Proc. R. Soc. London, Ser. A 375, 475 (1981).

[44] P. Honenberg, W. Kohn, Phys. Rev. B 136, 864 (1964).

[45] W. Kohn, L. J. Sham, Phys. Rev. A 140, 1133 (1965).

[46] A. D. Becke, Phys. Rev. A 38, 3098 (1988).

[47] A. D. Becke, J. Chem. Phys. 98, 5648 (1993).

[48] J. P. Perdew, Y. Wang, Phys. Rev. E 45, 13244 (1991).

[49] M. J. Frisch, G. W. Trucks, H. B.Schlegel, G. E. Scuseria, M. A. Robb, J. R. Cheeseman, J. A. Montgomery, T. Vreven, Jr., K. N. Kudin, J. C. Burant, J. M. Millam, S. S. Iyengar, J. Tomasi, V. Barone, B. Mennucci, M. Cossi, G. Scalmani, N. Rega, G. A. Petersson, H. Nakatsuji, M. Hada, M. Ehara, K. Toyota, R. Fukuda, J. Hasegawa, M. Ishida, T. Nakajima, Y. Honda, O. Kitao, H. Nakai, M. Klene, X. Li, J. E. Knox, H. P. Hratchian, J. B. Cross, V. Bakken, C. Adamo, J. Jaramillo, R. Gomperts, R. E. Stratmann, O. Yazyev, A. J. Austin, R. Cammi, C. Pomelli, J. W. Ochterski, P. Y. Ayala, K. Morokuma, G. A. Voth, P. Salvador, J. J. Dannenberg, V. G. Zakrzewski, S. Dapprich, A. D. Daniels, M. C. Strain, O. Farkas, D. K. Malick, A. D. Rabuck, K. Raghavachari, 


\section{Page 19 of 32

J. B. Foresman, J. V. Ortiz, Q. Cui, A. G. Baboul, S. Clifford, J. Cioslowski, B. B. Stefanov, G. Liu, A. Liashenko, P. Piskorz, I. Komaromi, R. L. Martin, D. J. Fox, T. Keith, M. A. Al-Laham, C. Y. Peng, A. Nanayakkara, M. Challacombe, P. M. W. Gill, B. Johnson, W. Chen, M. W. Wong, C. Gonzalez, J. A. Pople, Gaussian, Inc., Wallingford CT, (2004).

[50] C. P.Breneman, K. B. Wiberg, J. Comput. Chem. 11, 361 (1990).

[51] W. G. Hoover, Phys. Rev. A 31, 1695 (1985).

[52] DL_POLY is a parallel molecular dynamics simulation package developed at the Daresbury Laboratory Project for Computer Simulation under the auspices of the EPSRC for the Collaborative Computational Project for Computer Simulation of Condensed Phases (CCP5) and the Advanced Research Computing Group (ARCG) at the Daresbury Laboratory.

[53] E. Spohr, J. Chem. Phys. 107, 6342 (1997).

[54] J. Marti, G.Nau, M. C. Gordillo, E. Guardia, J. Chem. Phys. 124, 094703 (2006).

[55] A. Pertsin, M. Grunge, J. Chem. Phys. 125, 114707 (2006).

[56] J. G. Kirkwood, J. Chem. Phys. 7, 911 (1939).

[57] D. A. McQuarrie, Statistical Mechanics (Harper and Row, New-York, 1976).

[58] R. Mills, J. Phys. Chem. B 77, 685 (1973).

[59] A. K. Soper, M. G. Phillips, J. Chem. Phys. 107, 47 (1986).

[60] A. Luzar, M. G. Chandler, Phys. Rev. Lett. 76, 928 (1996).

[61] A. Chandra, S. Chowdhuri, J. Phys. Chem. B 106, 6779 (2002).

[62] H. Dominguez, M. L. Berkowitz, J. Phys. Chem. B 104, 5302 (2000).

[63] J. Janecek, R. R. Netz, Langmuir 23, 8417 (2007).

[64] R. S. Neves, A. J. Motheo, R. P. S. Fartaria, F. MS. S. Silva Fernandes, J. Electroanal. Chem. 612, 179 (2008). 
Table 1. Dimensions $\left(L_{x}, L_{y}, L_{z}\right)\left(\AA^{\circ}\right)$ of the simulation box, reciprocal lattice vectors $\left(\left|h_{x}^{\max }\right|,\left|h_{y}^{\max }\right|,\left|h_{z}^{\max }\right|\right)\left(\mathrm{A}^{\circ}-1\right)$, and the Ewald parameter $\alpha\left(\AA^{\circ}\right)$. The CPU times (hour) are reported for a production phase of 600 ps for water embedded between two parallel plates and a production phase of 300 ps and for the grafted NTA-Cu(II) ligands. The reported CPU time represents the real time of the job carried out over 8 processors.

Method

$$
L_{x} \quad L_{y} \quad L_{z}
$$

Water embedded between two graphite plates

$\begin{array}{cccccccccc}\text { HKE } & 39.4 & 38.4 & 50 & 4 & 4 & & 0.080926 & 2340 & 514 \\ \text { EW3D/EW3DC } & 39.4 & 38.4 & 190 & 7 & 7 & 34 & 0.17329 & 2340 & 39\end{array}$

Grafted NTA-Cu(II) onto graphite plates with charges distributed on the opposite surface

$\begin{array}{cccccccccc}\text { HKE } & 39.4 & 38.4 & 50 & 4 & 4 & & 0.080926 & 2200 & 330 \\ \text { EW3D/EW3DC } & 39.4 & 38.4 & 190 & 7 & 7 & 34 & 0.17329 & 2200 & 15\end{array}$

Grafted NTA-Cu(II) onto graphite plates with $\mathrm{Na}^{+}$counter-ions
EW3DC
$\begin{array}{lll}39.4 & 38.4 & 320\end{array}$
7
$7 \quad 58$
0.17329
3715
67 


\section{Page 21 of 32

Table 2. Lennard-Jones $\left(U_{L J}\right)$ and Coulombic $\left(U_{E L E C}\right)$ energy contributions $\left(\mathrm{kJ} \mathrm{mol}{ }^{-1}\right)$, overall coefficient diffusion $D$, parallel $(D / /)$ and perpendicular $\left(D_{\perp}\right)$ components of $D\left(10^{-5} \mathrm{~cm}^{2} \mathrm{~s}^{-1}\right)$. The diffusion coefficients are calculated over 60 time origins separated by $0.5 \mathrm{ps}$. The calculation is performed over a total time of $50 \mathrm{ps}$. In the case of the grafted $\mathrm{NTA}-\mathrm{Cu}(\mathrm{II})$ complexes, the diffusion coefficients are calculated at two different locations (zone $1 /$ zone 2). The zone 1 and zone 2 are defined by $0 \AA^{\circ}<z<25 \AA^{\circ}$ and $25 \AA^{\circ}<z<50 \AA^{\circ}$, respectively. The experimental self-diffusion coefficient $\left(10^{-5} \mathrm{~cm}^{2} \mathrm{~s}^{-1}\right)$ of water [58] at $298 \mathrm{~K}$ is reported for comparison.

\begin{tabular}{cccccc} 
Method & $U_{L J}$ & $U_{E L E C}$ & $D$ & $D_{/ /}$ & $D_{\perp}$ \\
\hline \multicolumn{5}{c}{ Water } \\
& & \multicolumn{4}{c}{ between graphite plates } \\
HKE & $1.736 \times 10^{4}$ & $-1.295 \times 10^{5}$ & 2.1 & 2.3 & 1.8 \\
EW3D & $1.722 \times 10^{4}$ & $-1.296 \times 10^{5}$ & 2.5 & 2.7 & 1.9 \\
EW3DC & $1.722 \times 10^{4}$ & $-1.296 \times 10^{5}$ & 2.6 & 3.0 & 1.8
\end{tabular}

Grafted NTA-Cu(II) complexes onto graphite plates with charges distributed on the opposite surface

$\begin{array}{cccccc}\text { HKE } & 1.802 \times 10^{4} & -1.450 \times 10^{5} & 0.9 / 2.4 & 0.8 / 3.0 & 0.9 / 1.9 \\ \text { EW3D } & 1.793 \times 10^{4} & -1.460 \times 10^{5} & 1.0 / 2.6 & 1.0 / 3.1 & 0.9 / 1.9 \\ \text { EW3DC } & 1.790 \times 10^{4} & -1.460 \times 10^{5} & 1.0 / 2.5 & 1.1 / 2.8 & 0.8 / 2.2\end{array}$

Experimental diffusion coefficient of water [58] $\quad 2.3$ 


\section{Figure captions}

Figure 1: $\quad$ a) Modified Nitrilotriacetic acid (NTA) complexed by $\mathrm{Cu}(\mathrm{II})$ cation b) atomic charges calculated from DFT/B3LYP method and c) snapshot of MD configuration of 10 grafted NTA-Cu(II) complexes onto the HOPG surface without the water molecules. Oxygen (red), Carbon (black), Nitrogen (blue), $\mathrm{Cu}$ (yellow), Hydrogen (white).

Figure 2: $\quad$ a) Water molecular density profiles along the $z$-direction for HKE (open circles), EW3D (solid lines) and EW3DC (dashed lines). b) Temperature profiles of $T(z)$ calculated from $1 / 3\left(T_{x x}(z)+T_{y y}(z)+T_{z z}(z)\right)$ for the three methods. c) O-O pair correlation functions for water in $1.0 \AA$ slab which is centered in the middle of the simulation cell. The diamond points correspond to the three dimensional radial distribution function calculated in a bulk water simulation cell.

Figure 3: $\quad$ a) Cumulative time average of the factor $G_{k}$ of TIP4P/2005 water model at $298 \mathrm{~K}$ as a function of the different methods as indicated in the legend.

b) Convergence of the square of the average total dipole moment for water. The inset shows the $M_{z}(z)$ profile along the $z$-direction for the three methods. $M_{z}$ is given in Debye $\left(1 \mathrm{D}=10^{-18} \mathrm{esu} \mathrm{cm}\right)$.

Figure 4: a) Molecular density profiles of water and atomic density profiles of the grafted molecules along the $z$-direction for HKE (open circles), EW3D (solid lines) and EW3DC (dashed lines). The density profiles of the grafted molecules are scaled down by 2 for clarity. b) Profiles of the number of hydrogen bonds between water molecules along the direction normal to the surfaces. c) O-O pair correlation functions for water in 1.0 A slab located at $z=30 \AA$.

Figure 5: a), $z$-component of the total dipole moment of the slab centered at $z$ along the $z$-direction; b) angular density profile of probability $n(\cos \theta)$ as a function of the cosinus of $\theta$ for three different zones $\mathrm{A}(20 \AA<z<$ $30 \AA), \mathrm{B}(40 \AA<z<50 \AA)$ and $\mathrm{C}(60 \AA<z<70 \AA)$. $\theta$ is formed by the dipole moment of water molecules and the $z$ - axis direction, represented by a unit vector perpendicular to the graphite surface; c) charge density and d) electric field profiles along the direction normal to the surface. 
Figure 6: $\quad$ Snapshot of a equilibrium configuration of 10 grafted NTA-Cu(II) complexes onto the HOPG surface with the water molecules. The water molecules are represented by stick-balls where the bigger balls represent the oxygen atom and smaller balls the hydrogen atoms. The sodium cations are represented in gray.

Figure 7: $\quad$ a) Density profiles of different atom types as a function of $z$ and b) density profiles of the hydrogen bond between the grafted molecule and the water molecules.

Figure 8: $\quad$ a) Parallel and perpendicular components of the mean-squared displacements and b) velocity autocorrelations functions of water molecules for two different zones: a grafted zone defined by $0 \AA<z<20 \AA$ and a bulklike zone defined by $35 \AA<z<80 \AA$.

Figure 9: a) Dispersive and electrostatic energy contributions between the grafted molecule of interest and the remaining 9 grafted molecules; b) Moleculewater energy contributions and number of water molecules interacting with the considered grafted molecule within two spheres of radii $12 \AA$ and $18 \AA$. 
a)

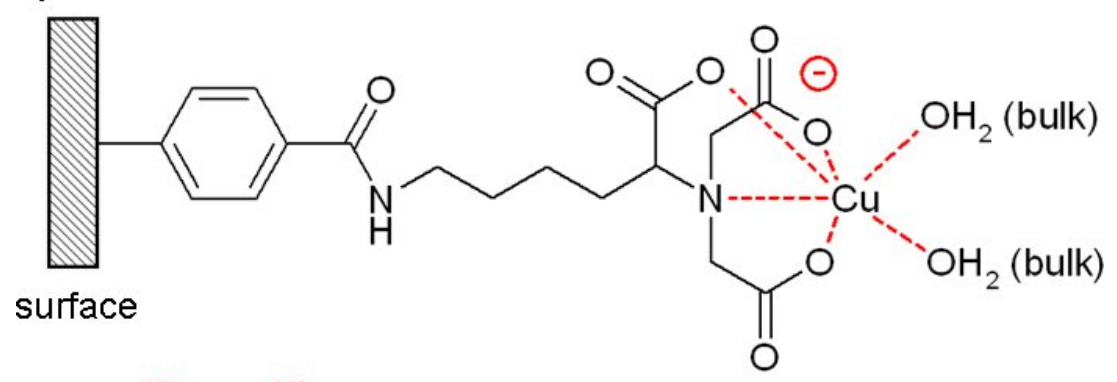

b)

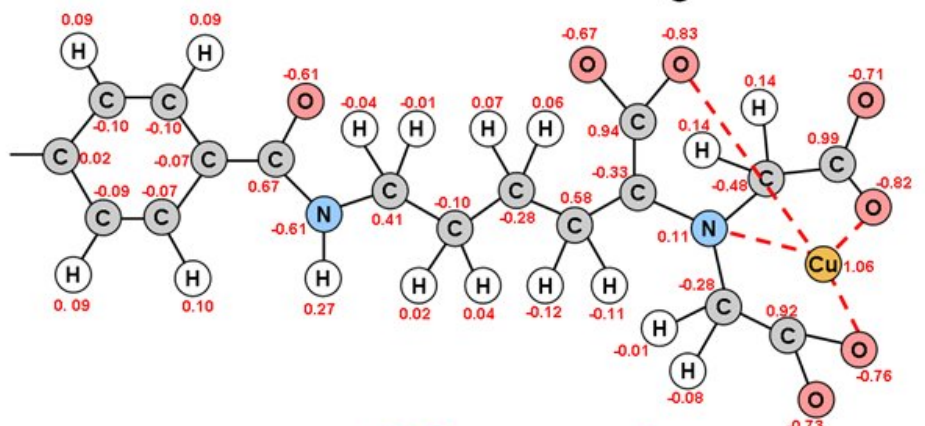

c)

Figure 1. 

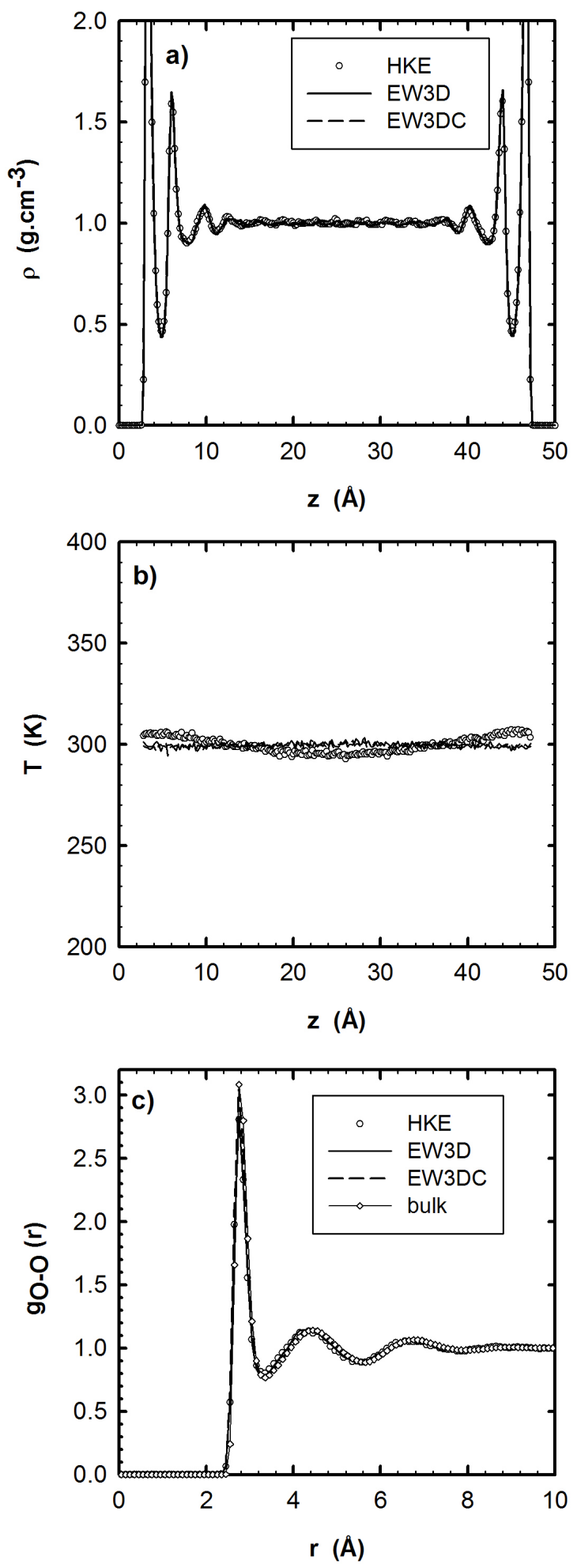

Figure 2 . 

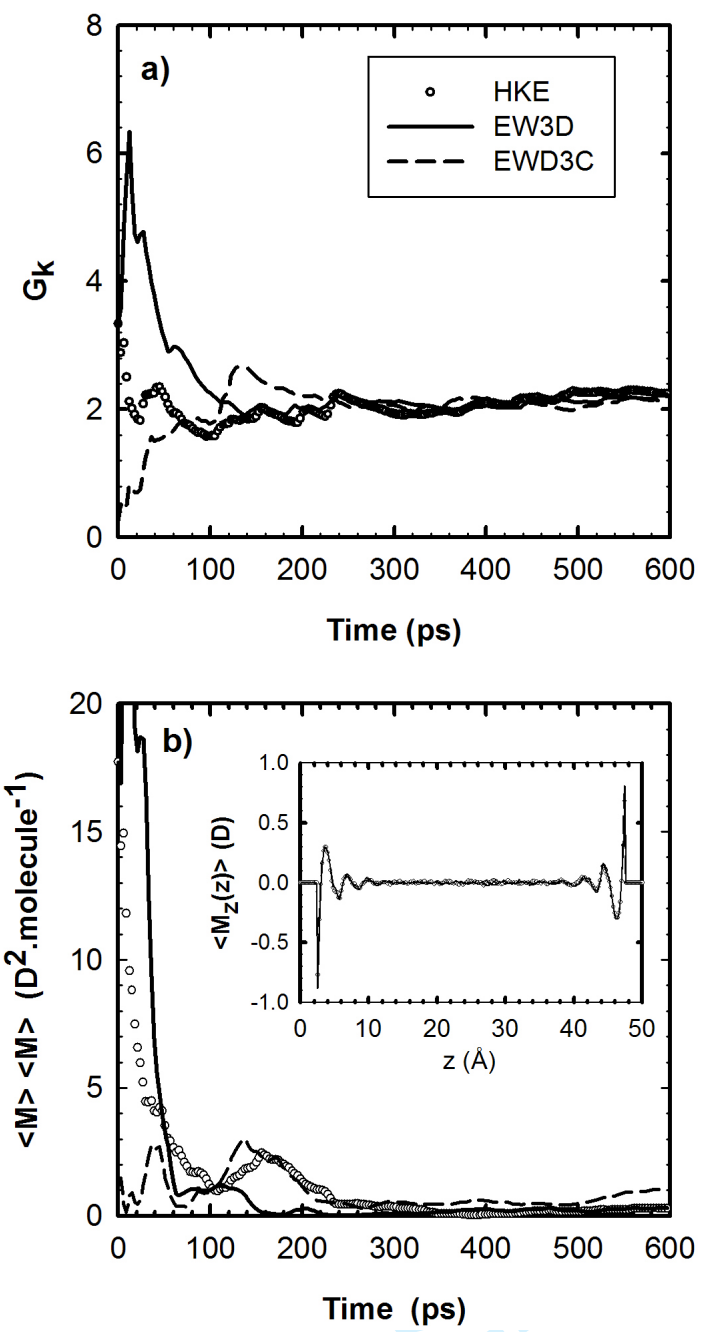

Figure 3. 


\section{Page 27 of $32 \quad$ Molecular Physics}
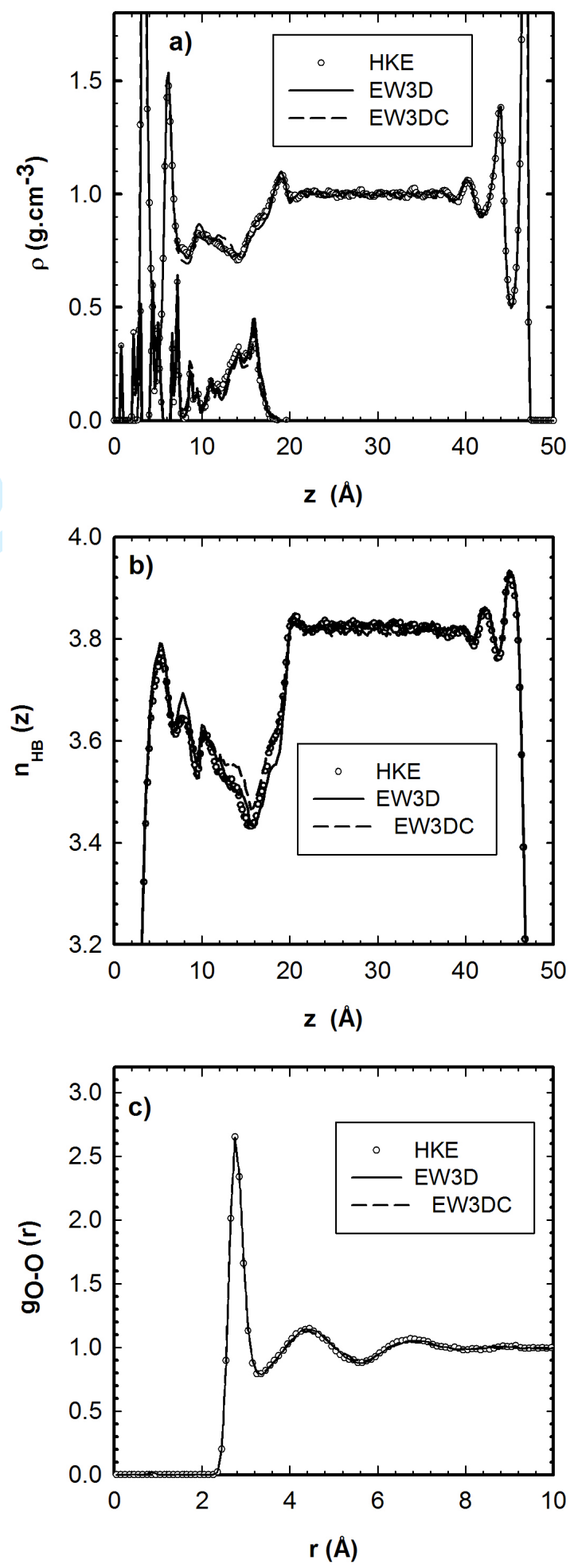

Figure 4 . 

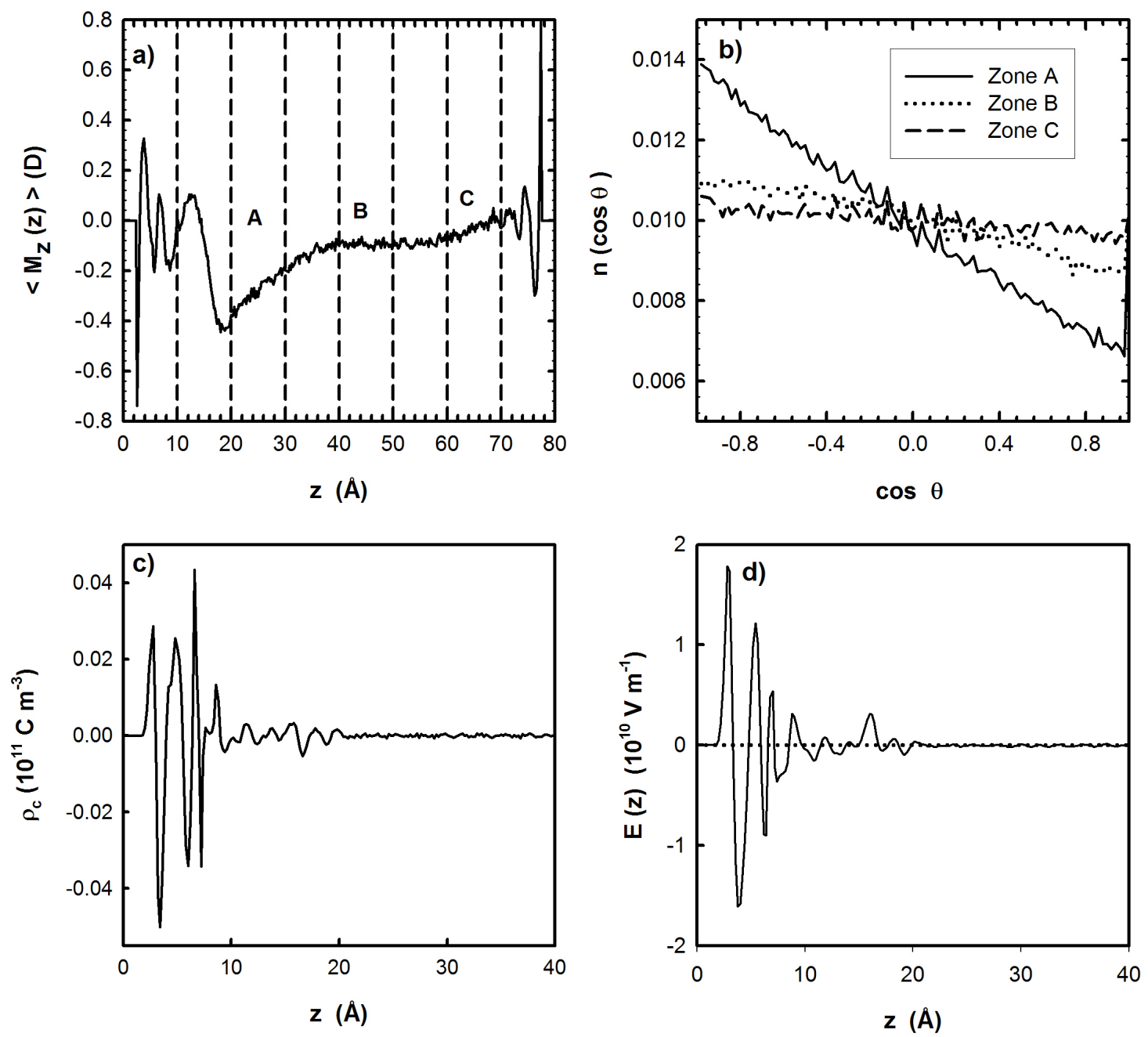

Figure 5. 


\section{Page 29 of 32

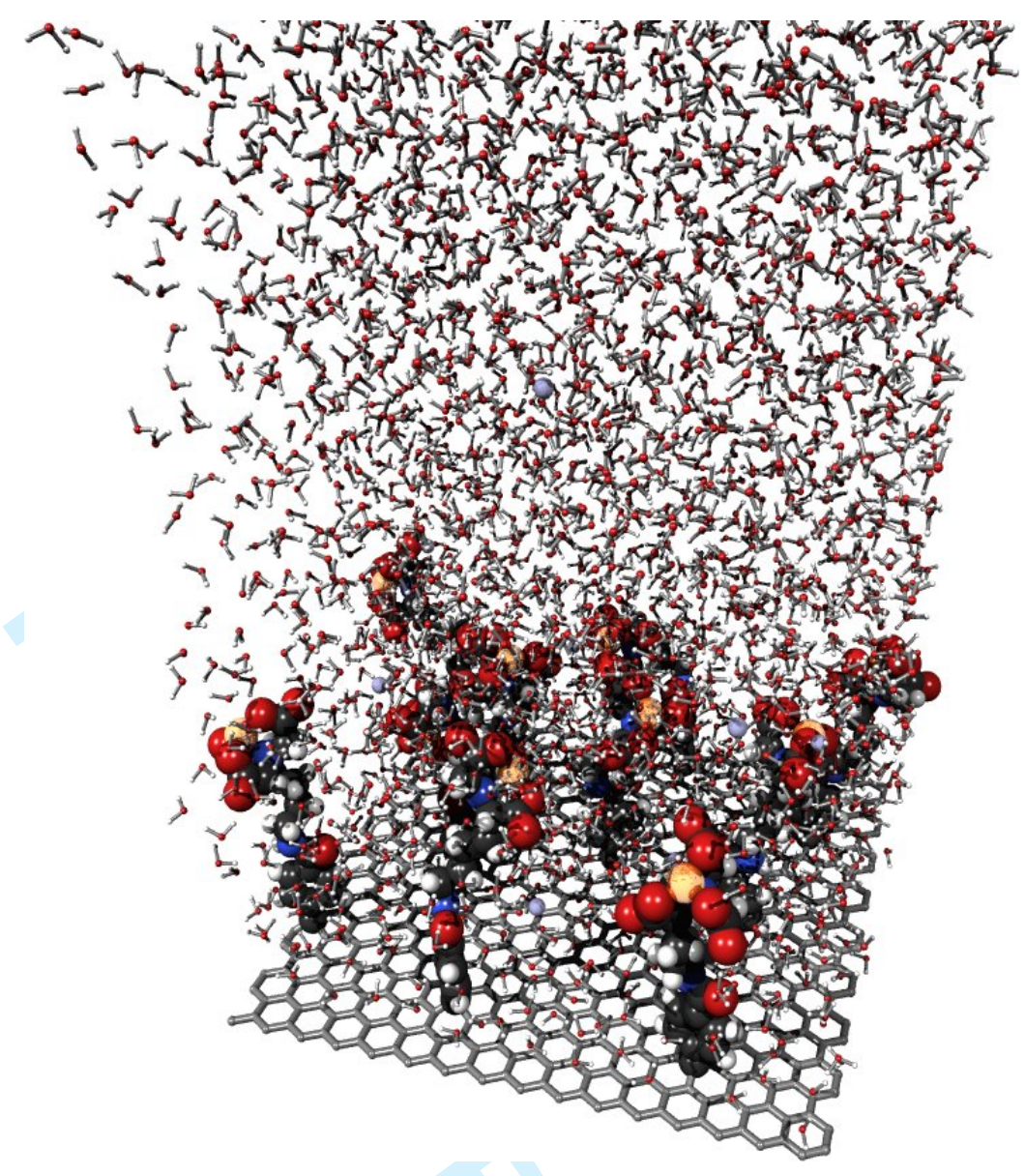

Figure 6. 

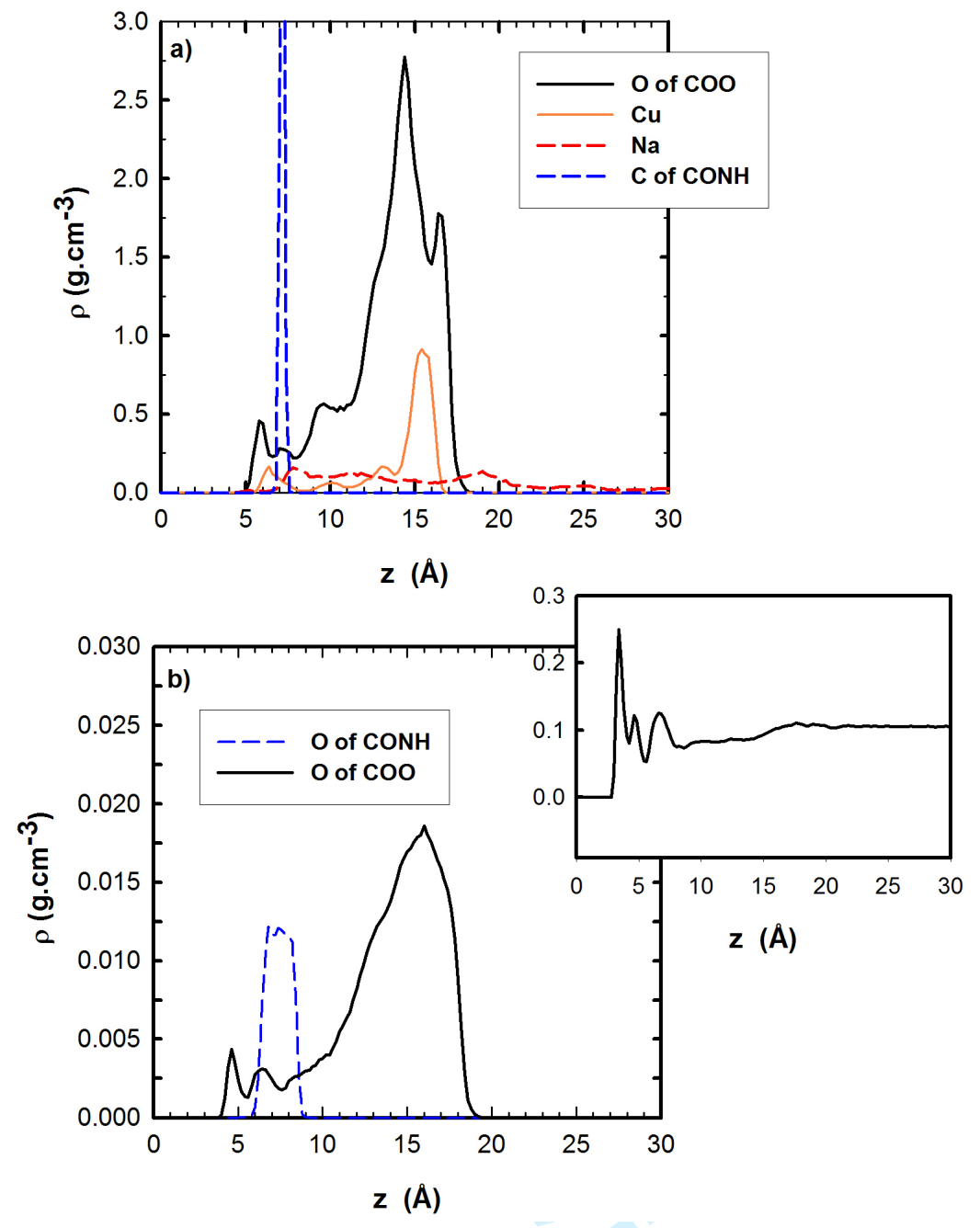

Figure 7. 


\section{Molecular Physics}
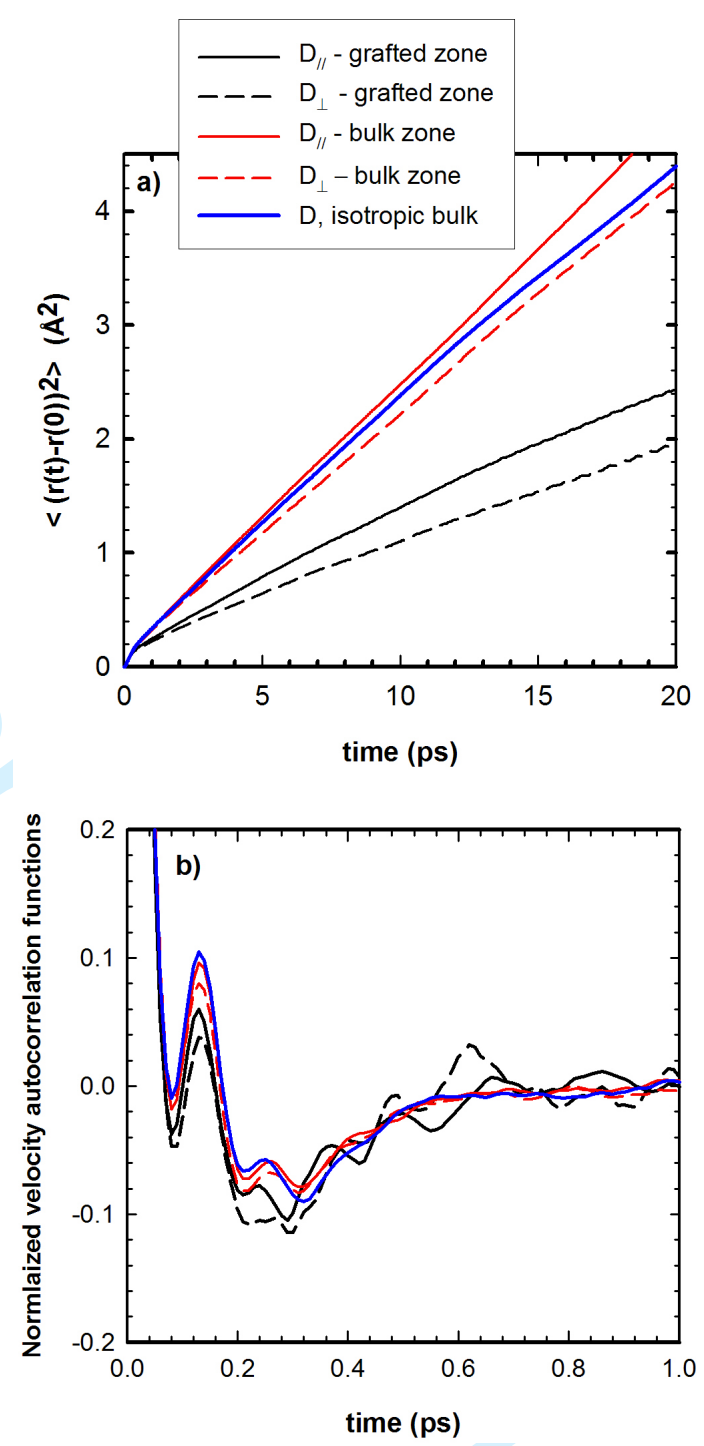

Figure 8 . 

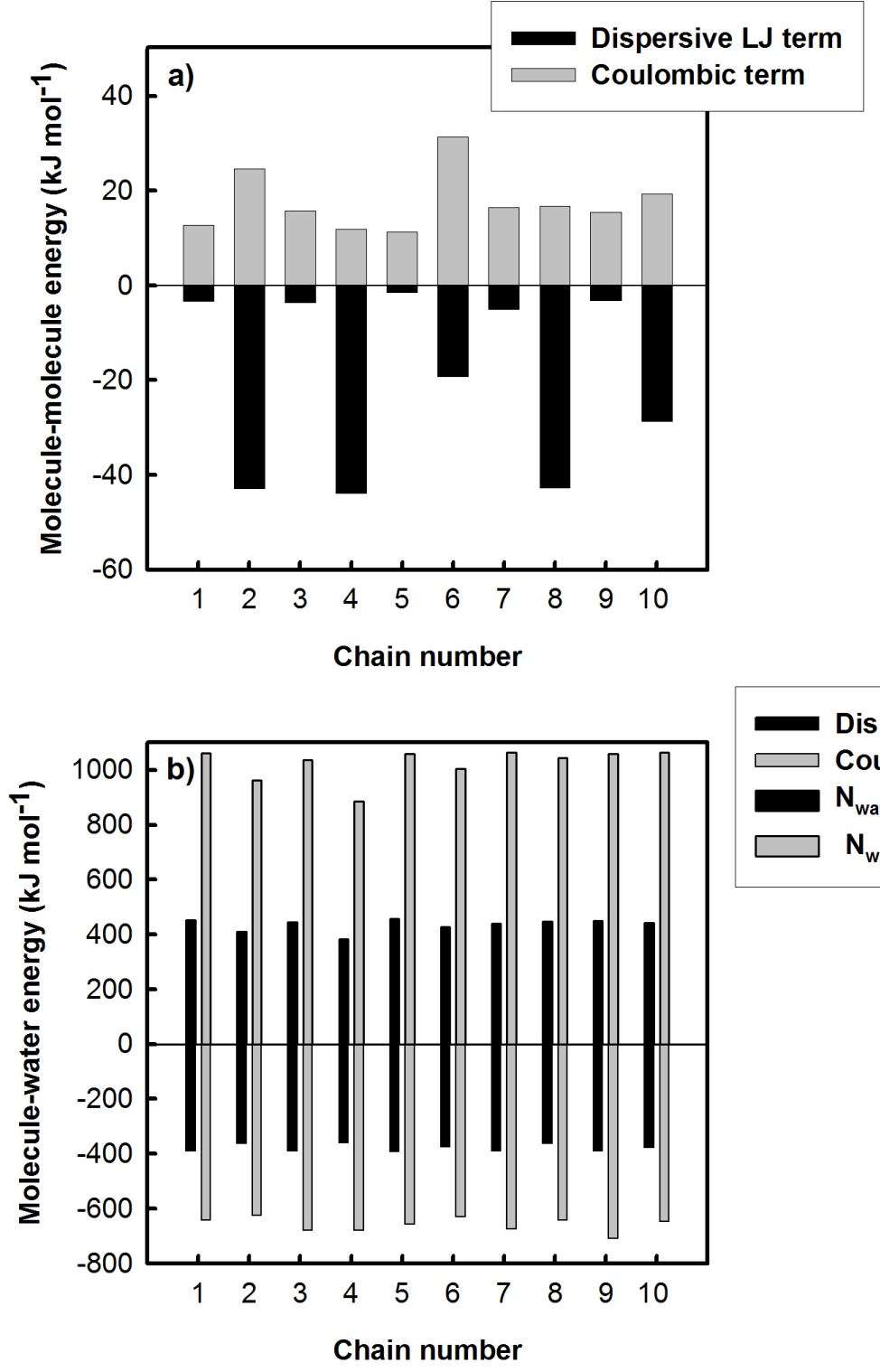

Dispersive LJ term $\square$ Coulombic term $N_{\text {water }}(12 A)$ $\square N_{\text {water }}(18 \mathrm{~A})$

Figure 9. 